\title{
Ein Cochrane Review zur Wirkung von Tanz-, Bewegungstherapie bei Demenz und zwei RCTs zur Wirkung von Gesellschaftstanz auf Kognition und Sturzhäufigkeit im Alter
}

\author{
Iris Bräuninger
}

D ieser Beitrag stellt die Ergebnisse eines aktuellen Cochrane Reviews zur Tanz-, Bewegungstherapie bei Demenz vor. Cochrane Reviews sind systematische Reviews auf höchstem Evidenzlevel, welche randomisierte kontrollierte Studien (RCTs) einschließen. Außerdem werden zwei RCTs zur Wirkung von Gesellschaftstanz auf Sturzhäufigkeit und Kognition im Alter vorgestellt, welche in Sydney, Australien, durchgeführt wurden.

\section{Cochrane Review zu Tanz-, Bewegungs- therapie bei Demenz}

Der Cochrane Review von Karkou und Meekums (2017) untersuchte das Potenzial von Tanz-, Bewegungstherapie bei Demenz und verglich die Behandlungseffekte mit Standardpflege oder anderen Behandlungsmöglichkeiten in Bezug auf Verhaltenssymptome und soziale, kognitive und emotionale Symptome. Verschiedene Formen der Tanzbewegungstherapie (z. B. Laban-basierte Tanz-, Bewegungstherapie, Chace-Tanz-, Bewegungstherapie oder Authentic Movement) sollten miteinander verglichen werden. Eingeschlossen wurden randomisierte kontrollierte Studien (RCTs) einschließlich Cross-over-Design und Cluster-RCTs mit Demenz-PatientInnen jeden Alters, welche Tanz-, Bewegungstherapie von ausgebildeten oder studentischen Tanz-, BewegungstherapeutInnen erhielten. Die Suche wurde in einer Reihe von Gesundheitsdatenbanken bis März 2016 durchgeführt. Darüber hinaus wurden graue Literaturquellen einbezogen, die Bibliographien relevanter Studien überprüft und internationale professionelle Verbände und ExpertInnen kontaktiert. Von 102 Studien, welche durch die Datenbanksuche und durch zusätzliche Quellen gefunden wurden, wurden letztendlich 19 Studien im

Volltext gelesen und überprüft. Keine der Studien entsprach den Einschlusskriterien. Die Autorinnen konnten keine Schlussfolgerungen ziehen, ob Tanz-, Bewegungstherapie bei Demenz wirksam sei oder nicht.

\section{Die Wirkung von Gesellschaftstanz auf Kognition und Sturzhäufigkeit im Alter}

Merom und KollegInnen (2016a) gingen von der Annahme aus, dass gleichgewichtsfördernde Aktivitäten wirksame Strategien in der Sturzprävention seien. Sie untersuchten in einem RCT die Wirkung von Gesellschaftstanz als Sturzprophylaxe bei älteren Erwachsenen und überprüften, ob sich die Anzahl der Stürze und die physischen und kognitiven sturzbezogenen Risikofaktoren durch Tanzinterventionen reduzieren ließen. Die Bewohnerlnnen von 23 Seniorenwohnanlagen in Sydney wurden zum Gesellschaftstanz eingeladen, der zweimal wöchentlich über 12 Monate stattfand (insgesamt 8oh). Teilnehmen konnten alle Bewohnerlnnen, welche eigenständig wohnten, mindestens $50 \mathrm{~m}$ selbständig zum Tanzraum gehen konnten und bereit waren, verschiedene Tests vor und nach den 12 Monaten durchzuführen. Von den 2,3\% Interessierten ( $N=530$, Durchschnittsalter 78 Jahre, $85 \%$ Frauen) wurden 12 Seniorenwohnanlagen mit 279 Teilnehmerlnnen auf die standardisierten Interventionsgruppen mit Gesellschaftstanz (Folk Dance oder Paartänze) und 11 Seniorenwohnanlagen mit 251 Teilnehmerlnnen auf die Wartekontrollgruppe randomisiert. Als Primärvariable wurde die Anzahl der Stürze innerhalb von 12 Monaten erfasst: Hierfür dokumentierten die TeilnehmerInnen beider Gruppen täglich im Monatskalender die Anzahl der Stürze und sandten jeweils am Monatsende 
den Kalender zurück. Eine weitere Primärvariable maß die Zeit, welche die Teilnehmerlnnen zum Ausfüllen des Trail Making Tests (TMT) benötigten. Die sekundären Ergebnisvariablen mit standardisierten Instrumenten erfassten das Sturzrisiko, die funktionelle Mobilität und gesundheitsbezogene Lebensqualität. Von den TeilnehmerInnen füllten $98 \%(n=522)$ die Daten zu Stürzen aus, und $80 \%$ $(\mathrm{n}=424)$ nahmen an dem Abschlusstest am Ende der 12-monatigen Intervention teil (80\%). An der Tanzintervention nahmen die Teilnehmerlnnen im Durchschnitt lediglich zu 51\% teil. Als Ergebnis konnte weder ein Effekt des Gesellschaftstanzes auf die Reduktion der Sturzhäufigkeit noch auf andere sturzbezogene Risikofaktoren wie Balance, Unterschenkelstärke, kognitive Verarbeitungsgeschwindigkeit oder Aufgabenstellung nachgewiesen werden. Lediglich die Ganggeschwindigkeit verbesserte sich geringfügig. Teilnehmerlnnen mit mehreren Stürzen im Vorjahr der Studie wiesen einen Anstieg der Stürze während der 12-monatigen Tanzintervention im Vergleich zur Kontrollgruppe auf. Als Schlussfolgerung raten die AutorInnen von unspezifischen Tanzinterventionen als Sturzprophylaxe ab. Stattdessen empfehlen sie ein modifiziertes Gesellschaftstanzprogramm, welches strukturierte Tanztrainingselemente inklusive Balanceübungen enthalten und zielgruppenorientiert (niedrige vs. hohe Risikogruppe) ausgerichtet sein sollte.

Der zweite RCT von Merom und KollegInnen (2016b) beschäftigte sich mit dem Effekt von Gesellschaftstanz und Walking auf die kognitiven Fähigkeiten von Erwachsenen im Alter von 60 und älter. Die Teilnehmerlnnen ( $\mathrm{N}=115)$ wurden zufällig auf eine Gesellschaftstanz-Intervention $(\mathrm{n}=60$; von den ursprünglich 60 randomisierten Tanzteilnehmerlnnen wechselten zwei zur Walking-Gruppe, und drei wechselten von der Walking Gruppe zur Tanzgruppe) und eine Walking-Intervention ( $n=55$; von den ursprünglich 55 randomisierten Walking-Teilnehmerlnnen wechselten drei zur Tanzgruppe und zwei von der Tanzgruppe zur Walkinggruppe) verteilt. Die Gruppen wurden vor und nach der achtmonatigen Intervention
(Baseline und delayed Baseline, 32 Wochen nach Baseline) in Hinblick auf die alterssensitiven kognitiven Domänen und in Hinblick auf Veränderungen der Trainingskapazität, sensomotorischen Fähigkeiten und kognitiven Veränderungen anhand standardisierter Tests miteinander verglichen. Das vereinheitlichte Tanzinterventionsprogramm beinhaltete für je eine Stunde zweimal wöchentlich Gesellschaftstänze (Rock and Roll, Foxtrott, Walzer, Salsa und Rumba) über acht Monate hinweg, welches von trainierten KursleiterInnen angeboten wurde (ca. 7oh). Die Kontrollgruppe erhielt ein Walking Selbsthilfe-Programm mit einem Schrittzähler und wurde aufgefordert, das Programm über eine Stunde zweimal wöchentlich und acht Monate lang zu praktizieren. Zusätzlich konnten die Kontrollgruppen-TeilnehmerInnen freiwillig einmal alle vierzehn Tage an Gruppentreffen in einem Gemeinschaftspark teilnehmen zur Unterstützung sozialer Kontakte. Die Ergebnisse zeigten keinen signifikanten Unterschied zwischen der Tanzintervention im Vergleich zur Walking Intervention in Bezug auf die alterssensitiven kognitiven Domänen, der Trainingskapazität oder sensomotorischen Fähigkeiten und kognitiven Veränderungen. Lediglich eine der kognitiven Domänen, das räumliche Gedächtnis, wurde in der Tanzgruppe verbessert.

\section{Schlussfolgerung}

Der Cochrane Review von Karkou und Meekums (2017) zeichnet sich durch höchste Qualitätskriterien aus. So wurden die Forschungsfragen klar definiert, die Suchstrategien und die Bewertung der Studien adäquat angewendet und von zwei Reviewern unabhängig voneinander durchgeführt. Das Ergebnis, dass bislang keine hochwertigen Studien zum Thema Tanz-, Bewegungstherapie und Demenz existieren, ist wichtig und sollte zukünftig als Rechtfertigung für die Dringlichkeit und die Begründung von Forschungsprojekten in diesem Bereich geltend gemacht werden. Beide RCTs von Merom und KollegInnen (2016a, b) weisen sich durch gute methodische Qualitäten wie 
Geheimhaltung der Zuordnung zur Vermeidung von Selektionsbias (concealment) und teilweiser Verblindung der Teilnehmenden und der Forschungsassistenten bei den Voruntersuchungen zur Gruppenzugehörigkeit (Merom et al. 2016b) bzw. der Forschungsassistenten in Bezug auf Therapielokalität (Merom et al. 2016a) aus. Merom und KollegInnen (2016a) konnten keinen Effekt des Gesellschaftstanzes auf die Reduktion der Sturzhäufigkeit und sturzbezogenen Risikofaktoren (2016a) nachweisen und führten als eine Limitation der Studie die Beteiligung von $51 \%$ an. Eine Beteilung der Hälfte sollte jedoch für eine ambulante Studie meines Erachtens nicht als niedrig interpretiert werden. Vielmehr sollte in zukünftigen Studien mit älteren Menschen der Tatsache Rechnung getragen werden, dass mit höheren Ausfallquoten zu rechen ist, was in einer a priori Poweranalyse, also vor Beginn der Studie, berücksichtigt werden muss. Der zweite RCT führte genau diese a priori Poweranalyse durch und gewann dadurch und dank Concealment eine starke Aussagekraft: Gesellschaftstänze scheinen keine
Verbesserung der kognitiven Leistungen zu bewirken. Zukünftige Studien mit älteren Menschen sollten auf den Erkenntnissen dieser drei Studien aufbauen, und die Erkenntnisse sollten in der bewegungsorientierten Praxis mit älteren Menschen berücksichtigt werden.

\section{Literatur}

Karkou, V., Meekums, B. (2017): Dance movement therapy for dementia. Cochrane Database of Systematic Reviews 2, https://doi.org/10.1002/14651858. cdo11022.pub2

Merom, D., Grunseit, A., Eramudugolla, R., Jefferis, B., Mcneill, J., Anstey, K.J. (2016b): Cognitive benefits of social dancing and walking in old age: the Dancing Mind randomized controlled trial. Frontiers in aging neuroscience 8, https://doi.org/10.3389/ fnagi.2016.00026

Merom, D., Mathieu, E., Cerin, E., Morton, R. L., Simpson, J. M., Rissel, C., Anstey, K. J., Sherrington, C., Lord, S. R., Cumming, R. G. (2016a). Social dancing and incidence of falls in older adults: a cluster randomised controlled trial. PLoS Medicine 13 (8), https://doi.org/10.1371/journal.pmed.1002112

\section{Die Autorin}

\section{Dr. Iris Bräuninger}

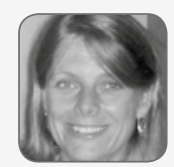

Dozentin an der Hochschule für Heilpädagogik Zürich (Studiengang Psychomotorik), ehemalige wissenschaftliche Mitarbeiterin bei Forschung \& Entwicklung und stellvertretende Leiterin Physio-, Tanz-, Bewegungstherapie \& Musiktherapie an der Psychiatrischen Universitätsklinik Zürich, Dozentin im Masterstudiengang Tanztherapie an der Autonomen Universität Barcelona (UAB),
Supervisorin, Ausbilderin und Lehrtherapeutin der deutschen und spanischen Berufsverbände (BTD, ADMTE), Kestenberg Bewegungsnotatorin, Psychotherapie (ECP), Private Praxis für Supervision und Therapie Bodensee.

Dr. Iris Bräuninger dancetherapy@mac.com oder iris.braeuninger@hfh.ch 